\title{
Effects of 22 novel CYP2D6 variants found in Chinese population on the metabolism of dapoxetine
}

This article was published in the following Dove Press journal:

Drug Design, Development and Therapy

15 February 2016

Number of times this article has been viewed

\section{Ren-ai $X u^{1, *}$ \\ Er-min $\mathrm{Gu}^{2}, *$ \\ Quan Zhou ${ }^{2}$ \\ Lingjing Yuan ${ }^{2}$ \\ Xiaoxia $\mathrm{Hu}^{2}$ \\ Jianping $\mathrm{Cai}^{3}$ \\ Guoxin $\mathrm{Hu}^{1,2}$}

'Department of Pharmacy, The First Affiliated Hospital of Wenzhou Medical University, ${ }^{2}$ Department of Pharmacology, School of Pharmacy, Wenzhou Medical University, Wenzhou, ${ }^{3}$ The Key Laboratory of Geriatrics, Beijing Hospital \& Beijing Institute of Geriatrics, Ministry of Health, Beijing, People's Republic of China

*These authors contributed equally to this work
Correspondence: Guoxin Hu The First Affiliated Hospital of Wenzhou Medical University, University-Town, Wenzhou 32500, Zhejiang, People's Republic of China

Fax +86 57786689983

Email hgx@wzmc.edu.cn

\section{Jianping Cai}

The Key Laboratory of Geriatrics, Beijing Hospital \& Beijing Institute of Geriatrics,

Ministry of Health, Beijing 100730,

People's Republic of China

Fax +86 I0 65237929

Email caijp6 I@vip.sina.com
Background: $C Y P 2 D 6$ is one of the most important members of the cytochrome P450 superfamily. Its genetic polymorphism significantly influences the efficacy and safety of some drugs, which might cause adverse effects and therapeutic failure.

Methods and results: The aim of this research was mainly to explore the catalytic activities of 22 newly reported CYP2D6 isoforms $(2 D 6 * 87, * 88, * 89, * 90, * 91, * 92, * 93, * 94, * 95$, *96, *97, *98, *R25Q, F164L, E215K, F219S, V327M, D336N, V342M, R344Q, R440C, R497C) on dapoxetine in vitro. The research was designed with an appropriate incubation system in test tubes and carried out in the constant temperature water. Through detecting its two metabolites desmethyldapoxetine and dapoxetine-N-oxide, the available data were obtained to explain the influence of CYP2D6 polymorphism on the substrate drug dapoxetine. As a result, the intrinsic clearance $\left(\mathrm{V}_{\max } / \mathrm{K}_{\mathrm{m}}\right)$ values of most variants were significantly altered when compared with the counterpart of CYP2D6*1, with most of these variants exhibiting either reduced $\mathrm{V}_{\max }$ and/or increased $\mathrm{K}_{\mathrm{m}}$ values. For dapoxetine demethylation pathway (which produces desmethyldapoxetine), $2 D 6 * 89$ and $E 215 \mathrm{~K}$ exhibited no markedly decreased relative clearance of $92.81 \%$ and $97.70 \%$, respectively. The relative clearance of rest 20 variants exhibited decrease in different levels, ranging from $20.44 \%$ to $90.90 \%$. For the dapoxetine oxidation pathway (which produces dapoxetine-N-oxide), the relative clearance values of three variants, $2 D 6^{*} 90$, *94, and $V 342 \mathrm{M}$, exhibited no markedly increased relative clearance of $106.17 \%, 107.78 \%$, and $109.98 \%$, respectively; the rest 19 variants exhibited significantly decreased levels ranging from $27.56 \%$ to $84.64 \%$. In addition, the kinetic parameters of two CYP2D6 variants (2D6*92 and $2 D 6 * 96)$ could not be detected, due to the defect of the CYP2D6 gene.

Conclusion: As the first report of all aforementioned alleles for dapoxetine metabolism, these data may help in the clinical assessment of the metabolic elimination of dapoxetine and may provide fundamental information for further clinical studies.

Keywords: genetic polymorphism, CYP2D6 variants, drug metabolism, dapoxetine, personalized treatment

\section{Introduction}

Polymorphism in the cytochrome P450 (CYP) family may have the most impact on the metabolism of therapeutic drugs. CYP2D6, CYP2C9, CYP2C19, and CYP3A4 polymorphisms account for the most frequent variations in Phase I metabolism of drugs, since almost $80 \%$ of drugs are metabolized by these enzymes. ${ }^{1}$ CYP2D6 is one of the highly polymorphic enzymes and participates in the metabolism of many therapeutic drugs that are commonly used in clinic, including the antidepressants fluoxetine, amitriptyline, and venlafaxine; the antitussive dextromethorphan; the 
$\beta$-adrenergic antagonists bufuralol and metoprolol; ${ }^{2}$ the opioid analgesics codeine, dihydrocodeine, and tramadol; ${ }^{3}$ the antipsychotic agent risperidone; ${ }^{4}$ and the selective serotonin reuptake inhibitor dapoxetine. ${ }^{5}$ CYP2D6 polymorphisms can lead to no enzyme expression (poor metabolism), very low enzyme activity (intermediate metabolism), or typically associated with overexpression of a metabolic enzyme (ultrarapid metabolism). ${ }^{6}$ These may cause adverse effects and therapeutic failures, thus more attention should be paid to the CYP2D6 polymorphism.

According to the National Center for Biotechnology Information website report, 370 variable sites of $C Y P 2 D 6$ have been found and more than 100 CYP2D6 alleles have been identified and named by the Human CYP Allele Nomenclature Committee (http://www.cypalleles.ki.se/cyp2d6.htm). In 2013, Qian et $\mathrm{al}^{7}$ analyzed the CYP2D6 gene of 2,129 unrelated healthy Chinese volunteers and detected 165 mutated sites, of which 67 sites were discovered for the first time. Among these, 22 novel mutation sites were nonsynonymous, and of them 12 mutation sites were named as *87-*93,*94A, $* 94 B$, and *95-*98 by the Human CYP Allele Nomenclature Committee. ${ }^{7}$ In later study, 22 newly reported CYP2D6 isoforms were transiently expressed to assess the enzymatic activity of these variants on dextromethorphan and bufuralol. ${ }^{8,9}$

Premature ejaculation (PE) is a common problem, with a global prevalence estimated to be $20 \%-40 \%{ }^{10}$ and has significant impact not only on the sufferer, but also on the partner, in terms of self-esteem, interpersonal distress, and sexual satisfaction. ${ }^{11}$ Dapoxetine, a selective serotonin reuptake inhibitor, is used for the treatment of PE in men aged 18-64 years. ${ }^{12}$ However, dapoxetine has numerous adverse effects like nausea, diarrhea, dizziness, insomnia, and nasopharyngitis. ${ }^{13-15}$ In addition, dapoxetine has no pharmacokinetic interactions with food, alcohol, or phosphodiesterase type 5 (PDE5) inhibitors. ${ }^{16,17}$ Dapoxetine is primarily metabolized by CYP3A4 and CYP2D6. ${ }^{5}$ Thus, the exploration of CYP3A4 or CYP2D6 gene polymorphisms on dapoxetine metabolism can be meaningful. In this study, we focus on the catalytic activities of 24 CYP2D6 isoforms on dapoxetine metabolism in vitro by detecting its two metabolites generated by CYP2D6 enzyme (shown in Figure 1). We hope these findings can provide reference for rational administration of drugs in the clinic and promote the development of personalized medicine.

\section{Materials and methods Chemicals and materials}

Dapoxetine, desmethyldapoxetine, dapoxetine-N-oxide, and carbamazepine were obtained from Sigma-Aldrich (St Louis, MO, USA). The UPLC ${ }^{\circledR}$ BEH C18 column $(2.1 \mathrm{~mm} \times 50 \mathrm{~mm}$, $1.7 \mu \mathrm{m}$ ) was obtained from the Waters (Ireland). Nicotinamide adenine dinucleotide phosphate was obtained from Promega (Madison, WI, USA). Formic acid (analytical reagent grade) was purchased from Sigma-Aldrich. Ultrapure water was obtained from a Milli-Q system (Millipore, Bedford, MA, USA). Liquid chromatography grade methanol and acetonitrile were purchased from Merck Chemicals Co., Ltd. (Darmstadt, Germany). Other chemicals and solvents were of analytical grade from Chemical Industries (Beijing, People's Republic of China).
A<smiles>CN(C)[C@H](CCOc1cccc2ccccc12)c1ccccc1</smiles>

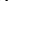

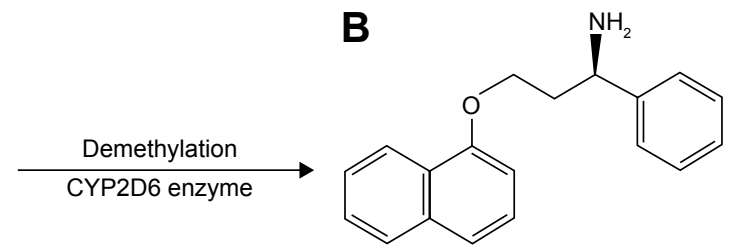

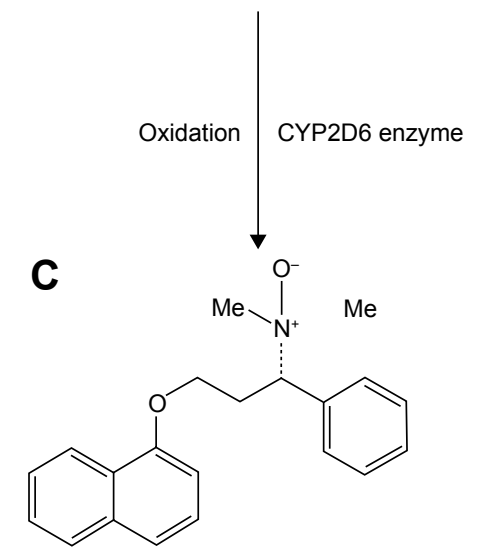

Figure I Structures of the analytes and Phase I metabolic pathway of dapoxetine by CYP2D6 enzyme. Notes: (A) Dapoxetine hydrochloride. (B) Desmethyldapoxetine. (C) Dapoxetine- $\mathrm{N}$-oxide. 


\section{Instrumentation}

Samples were analyzed by the ultra-performance liquid chromatography tandem mass-spectrometry with ACQUITY UPLC H-Class and XEVO TQD triple-quadrupole mass spectrometer (Waters Corp., Milford, MA, USA) equipped with an electrospray ionization interface. MassLynx 4.1 software (Waters Corp.) was used to control the instrument and process all the data of the samples.

\section{Incubation conditions}

Recombinant human CYP2D6 enzymes (the wild-type CYP2D6*1 and 24 CYP2D6 variants) generated in Spodoptera frugiperda 21 insect cells were obtained according to the previously reported method. ${ }^{8}$ The incubation mixture as the final assay concentration included $176.6 \mu \mathrm{L} 100 \mathrm{mmol} / \mathrm{L}$ potassium phosphate buffer (pH 7.4), 5 pmol wild-type CYP2D6*1 or other CYP2D6 recombinant variants, 5 pmol purified cytochrome b5, and $1.71 \mu \mathrm{L}$ of dapoxetine, which was made to the total volume of $200 \mu \mathrm{L}$. Dapoxetine was initially prepared in methanol solution and the concentration range was adjusted from 25 to $3,200 \mu \mathrm{M}$. The total concentration of methanol in the mixture was $<0.5 \%$. After incubating for 5 minutes, $1 \mathrm{mM}$ nicotinamide adenine dinucleotide phosphate-regenerating system (1.3 mM NADP $+3.3 \mathrm{mM}$ glucose-6-phosphate, $3.3 \mathrm{mM} \mathrm{MgCl}$, and 0.4 unit/mL glucose-6-phosphate dehydrogenase) was added. The mixture was incubated at $37^{\circ} \mathrm{C}$ for 40 minutes. In addition, the experiments were carried out in individual tubes and kept parallel, one for each concentration point. The reaction in tubes was terminated by cooling to $-80^{\circ} \mathrm{C}$ immediately. Then $400 \mu \mathrm{L}$ acetonitrile and $40 \mu \mathrm{L}$ internal standard carbamazepine were added. Carbamazepine was dissolved in acetonitrile solution at a concentration of $1 \mu \mathrm{g} / \mathrm{mL}$. After vortexing for 2 minutes and centrifuging at $13,000 \mathrm{rpm}$ for 10 minutes in $4^{\circ} \mathrm{C}$ environment, the supernatant of each tube was 1:1 diluted with ultrapure water and $2 \mu \mathrm{L}$ was injected into the ultra-performance liquid chromatography tandem mass-spectrometry system. The data were analyzed using SPSS Version 13.0 (SPSS Inc., Chicago, IL, USA). All the experimental results were as mean \pm standard deviation (SD) of three parallel measurements. All experiments were approved by the Ethics Committee of Beijing Hospital.

\section{Chromatographic conditions}

The liquid chromatographic separations were performed on an UPLC BEH C18 column $(2.1 \mathrm{~mm} \times 50 \mathrm{~mm}, 1.7 \mu \mathrm{m})$, with an inline $0.2 \mathrm{~mm}$ stainless steel frit filter connected to it. The column temperature was kept at $40^{\circ} \mathrm{C}$ constantly, while the samples in the autosampler room were maintained at $4^{\circ} \mathrm{C}$ with an injection volume of $2 \mu \mathrm{L}$ for detection. The initial mobile phase consisted of acetonitrile and water (containing 0.1\% formic acid) at a flow rate of $0.4 \mathrm{~mL} / \mathrm{min}$. Elution was in a linear gradient as follow: the acetonitrile content changing from $30 \%$ to $85 \%$ between 0 and 1 minute, maintained at $85 \%$ for 1 minute, then decreased to $30 \%$ within 12 seconds. The total run time of the analytes was 3 minutes. After each injection, the sample manager underwent a needle wash process, including a strong wash (methanol-water, 50/50, v/v) and a weak wash (methanol-water, 10/90, v/v). With aforementioned appropriate conditions, the retention times of desmethyldapoxetine, dapoxetine-N-oxide, dapoxetine, and carbamazepine were at $1.40,1.45,1.50$, and 1.23 minutes, respectively.

\section{Mass spectrometric conditions}

A Waters XEVO TQD triple-quadrupole mass spectrometer, equipped with an electrospray ionization source, was set to positive electrospray ionization in multiple reactionmonitoring mode. Nitrogen was used as the desolvation gas $(600 \mathrm{~L} / \mathrm{h})$ and cone gas $(50 \mathrm{~L} / \mathrm{h})$. The selected ion monitoring conditions were defined as follows: capillary voltage $2.5 \mathrm{kV}$; source temperature $150^{\circ} \mathrm{C}$; and desolvation temperature $500^{\circ} \mathrm{C}$. The multiple reaction-monitoring mode of transitions as quantitative analysis were $\mathrm{m} / \mathrm{z} 306.0 \rightarrow 261.1, \mathrm{~m} / \mathrm{z}$ $292.0 \rightarrow 261.1, \mathrm{~m} / z$ 322.0 $\rightarrow 261.1$, and $\mathrm{m} / \mathrm{z} 237.0 \rightarrow 194.1$ for dapoxetine, desmethyldapoxetine, dapoxetine-N-oxide, and IS, respectively. The collision energy was set at $25 \mathrm{~V}$ for dapoxetine, desmethyldapoxetine, and dapoxetine-N-oxide, while $20 \mathrm{~V}$ for carbamazepine; the cone voltage was set at $50 \mathrm{~V}$ for dapoxetine, desmethyldapoxetine, and dapoxetine$\mathrm{N}$-oxide, while $40 \mathrm{~V}$ for carbamazepine, respectively.

\section{Statistical analysis}

The kinetic parameters $\left(\mathrm{K}_{\mathrm{m}}\right.$ and $\left.\mathrm{V}_{\max }\right)$ were performed by nonlinear regression curve fitting using the computer program Prism version 5 (GraphPad Software Inc., San Diego, CA, USA). Oneway analysis of variance was used for intergroup comparison. Dunnett's test was used to analyze differences in catalytic activity between $C Y P 2 D 6^{*} 1$ and other CYP2D6 mutants. Kinetic data for each variant were presented as the mean \pm SD of three independent experiments. Statistical analysis was performed using SPSS version 17.0 (SPSS Inc., Chicago, IL, USA), with $P<0.05$ considered statistically significant.

\section{Results}

From the aforementioned study, the catalytic activities of the wild-type and 24 allelic variants of CYP2D6 were assessed, using dapoxetine as the substrate drug. Michaelis-Menten plots of the tested 25 CYP2D6 enzymes are shown in Figures 2 and 3 , and corresponding kinetic parameters are summarized in 

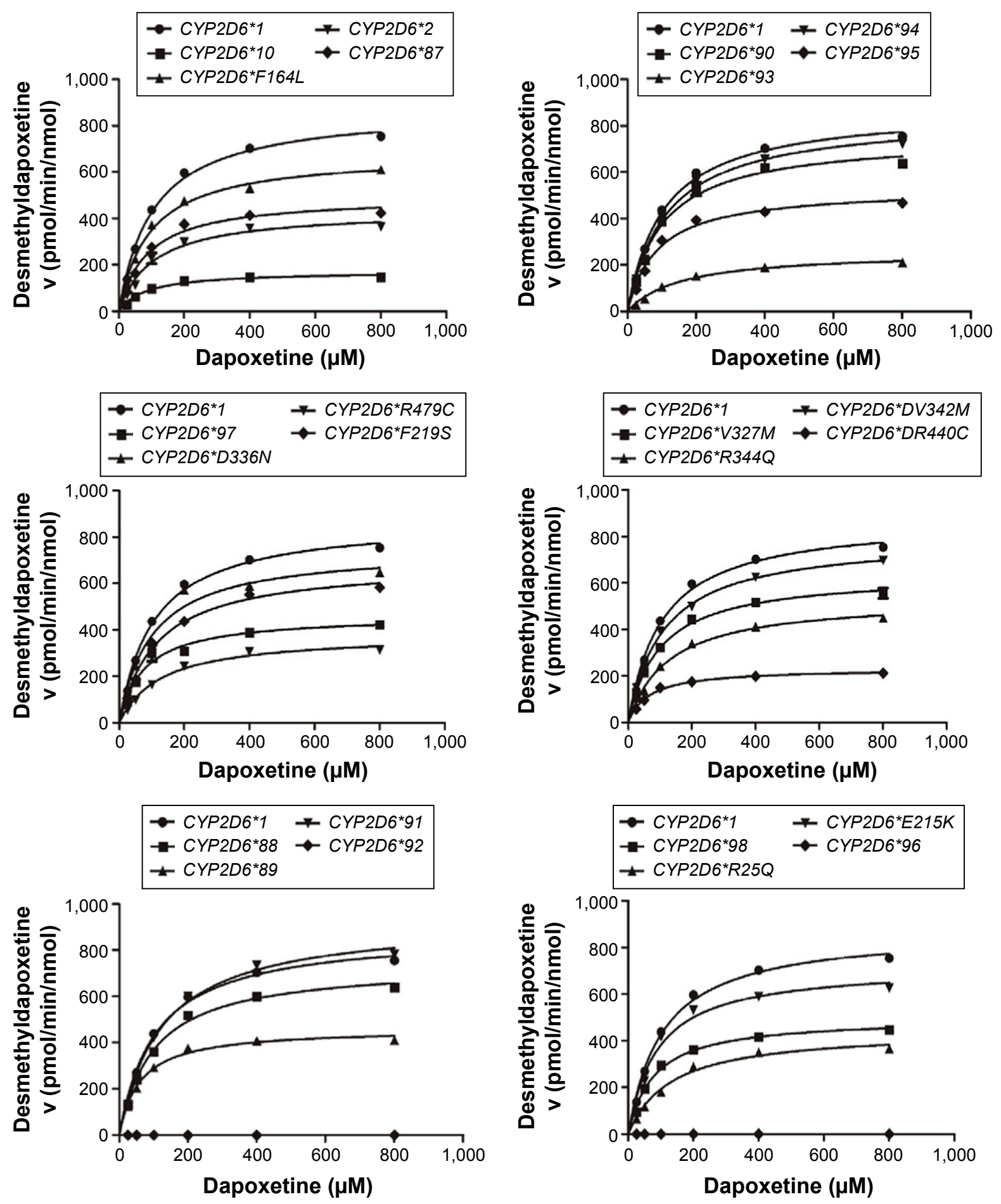

Figure 2 Michaelis-Menten curves of the enzymatic activity of the wild-type and 24 variants toward dapoxetine demethylation (each point represents the mean \pm standard deviation of three parallel experiments).

Note: The allelic variants with designated allele names have been properly arranged into six groups.

Tables 1 and 2. The intrinsic clearance $\left(\mathrm{V}_{\text {max }} / \mathrm{K}_{\mathrm{m}}\right)$ values of dapoxetine were significantly altered in most of the allelic variants, except occasionally a few, according to the analysis of its two metabolites. The relative clearance graph (compared with that of $2 D 6^{*} 1$ ) of dapoxetine demethylation and oxidation is shown in Figure 4.
The results of CYP2D6 allelic variants on dapoxetine demethylation are represented in Figure 2 and Table 1. Twelve of the allelic variants exhibited obvious reduced $\mathrm{V}_{\max }$ values when compared with $2 D 6^{*} 1$ (count as $100 \%$ ): three mutants $\left(2 D 6^{*} 10, * 93\right.$, and $\left.R 440 C\right)$ obviously reduced to $19.77 \%-29.41 \%$; nine variants $(2 D 6 * 2, * 87$, 

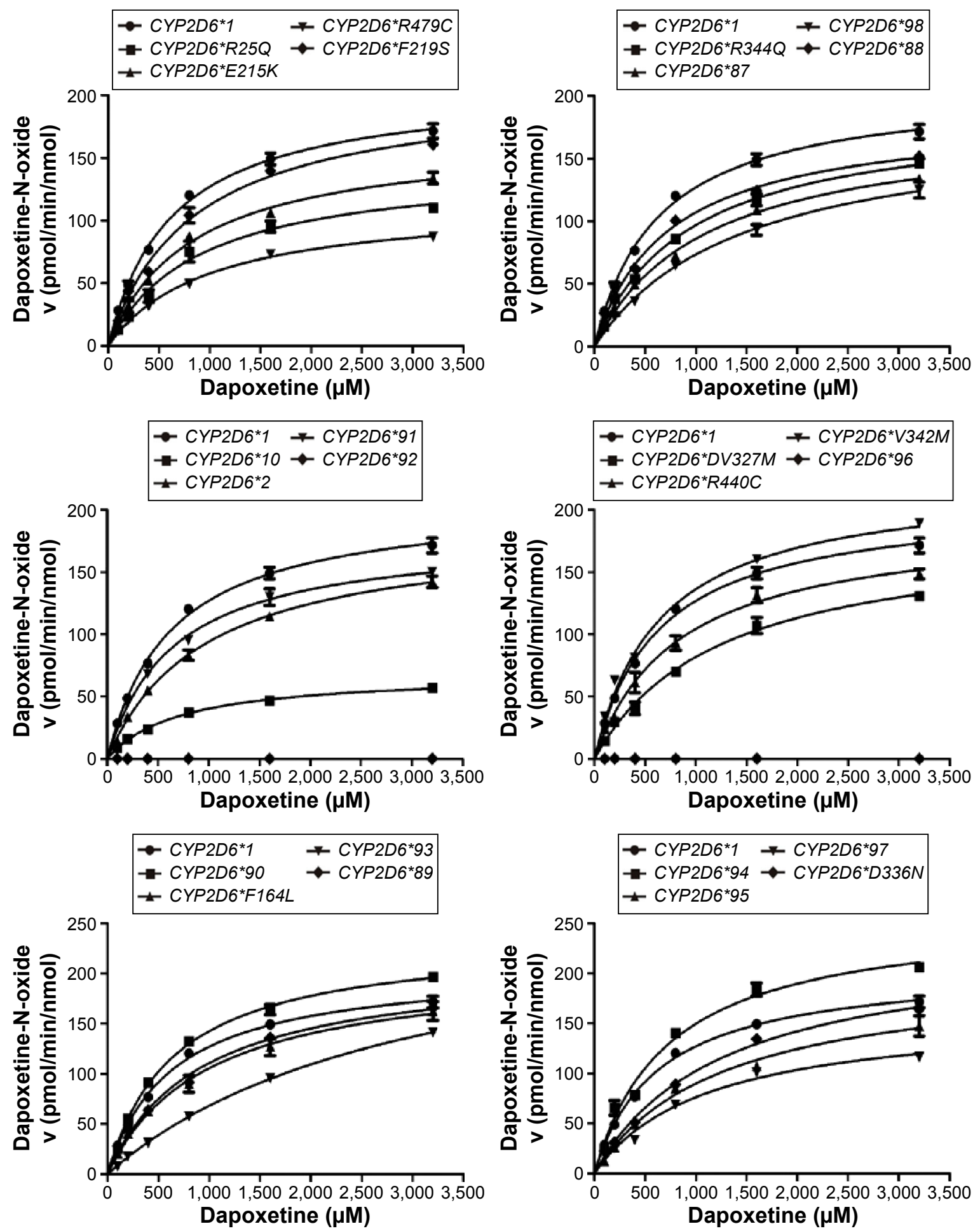

Figure 3 Michaelis-Menten curves of the enzymatic activity of the wild-type and 24 variants toward dapoxetine oxidation (each point represents the mean \pm standard deviation of three parallel experiments).

Note: The allelic variants with designated allele names have been properly arranged into six groups.

$* 89, * 95, * 97, * 98, R 25 Q, R 344 Q$, and $R 497 C$ ) decreased to $43.64 \%-61.11 \%$. Moreover, most variants showed no obvious changes on $\mathrm{K}_{\mathrm{m}}$ values: eight variants $(2 D 6 * 2, * 88$, *90, F164L, F219S, V327M, D336N, and V342M) showed no statistical difference; six variants $\left(2 D 6^{*} 10, * 87, * 95\right.$,
*97, *98, and $E 215 K)$ mildly decreased to $78.40 \%-84.22 \%$; six variants $(2 D * * 91, * 93, * 94, R 25 Q, R 344 Q$, and $R 497 C)$ lightly increased to $108.57 \%-123.40 \%$. Except two variants $(2 D 6 * 89$ and $R 440 C)$, their $\mathrm{K}_{\mathrm{m}}$ values decreased to $56.58 \%$ and $62.08 \%$, respectively. As a result, $\mathrm{V}_{\max } / \mathrm{K}_{\mathrm{m}}$ values for 
Table I Kinetic parameters from demethylation by recombinant wild-type and 24 CYP2D6 allelic variants on dapoxetine

\begin{tabular}{|c|c|c|c|c|c|}
\hline Variants & $\begin{array}{l}\text { Allele frequency } \\
\text { (\%) }\end{array}$ & $\begin{array}{l}V_{\max } \\
(\mathrm{pmol} / \mathrm{min} / \mathrm{nmol} \text { of } \mathrm{P} 450)\end{array}$ & $\mathbf{K}_{\mathrm{m}}(\mu \mathrm{M})$ & $\begin{array}{l}\text { Intrinsic clearance } \\
\left(\mathbf{V}_{\max } / K_{m}\right)\end{array}$ & $\begin{array}{l}\text { Relative clearance } \\
\text { (\% of wild-type) }\end{array}$ \\
\hline $2 D 6 * 1$ & 26.56 & $875.97 \pm 2.78$ & $105.67 \pm 1.24$ & $8.29 \pm 0.07$ & 100.00 \\
\hline 2D6*2 (R296C; S486T) & 10.34 & $435.23 \pm 11.15^{\#}$ & $106.35 \pm 15.08$ & $4.14 \pm 0.45^{\#}$ & 49.63 \\
\hline 2D6*10 (P34S; S486T) & 42.86 & $173.17 \pm 2.47^{\#}$ & $82.84 \pm 8.21^{\#}$ & $2.10 \pm 0.22^{\#}$ & 25.31 \\
\hline 2D6*87 (A5V) & 0.023 & $495.90 \pm 7.22^{\#}$ & $87.53 \pm 1.23^{\#}$ & $5.67 \pm 0.10^{\#}$ & 68.37 \\
\hline 2D6*88 (VI04A) & 0.094 & $738.30 \pm 2.42^{\#}$ & $101.05 \pm 1.19$ & $7.31 \pm 0.09^{\#}$ & 88.12 \\
\hline 2D6*89 (LI L2S) & 0.023 & $459.87 \pm 4.53^{\#}$ & $59.79 \pm 0.74^{\#}$ & $7.69 \pm 0.04$ & 92.81 \\
\hline $2 D 6 * 90(K / 47 R)$ & 0.047 & $752.30 \pm 2.69^{\#}$ & $101.71 \pm 7.36$ & $7.42 \pm 0.50^{\#}$ & 89.55 \\
\hline $2 \mathrm{D6} 691$ (CI6IS) & 0.023 & $934.33 \pm 11.67^{\#}$ & $124.10 \pm 6.18^{\#}$ & $7.54 \pm 0.28^{\#}$ & 90.90 \\
\hline 2D6*92 (2 I8frameshift) & 0.023 & ND & ND & ND & ND \\
\hline $2 D 6 * 93(T 249 P)$ & 0.023 & $257.60 \pm 1.56^{\#}$ & $152.17 \pm 2.98^{\#}$ & $1.69 \pm 0.03^{\#}$ & 20.44 \\
\hline 2D6*94 (D337G) & 0.164 & $842.97 \pm 2.43^{\#}$ & $114.73 \pm 1.99^{\#}$ & $7.35 \pm 0.1 I^{\#}$ & 88.66 \\
\hline $2 \mathrm{D6} * 95(\mathrm{R388H})$ & 0.047 & $531.20 \pm 10.15^{\#}$ & $87.43 \pm 5.4 I^{\#}$ & $6.09 \pm 0.25^{\#}$ & 73.39 \\
\hline 2D6*96 (424STOP) & 0.074 & ND & ND & ND & ND \\
\hline 2D6*97 (F457L) & 0.047 & $462.97 \pm 4.63^{\#}$ & $77.76 \pm 8.39 \#$ & $6.00 \pm 0.62^{\#}$ & 72.34 \\
\hline 2D6*98 (H463D) & 0.023 & $497.17 \pm 5.66^{\#}$ & $78.70 \pm 4.43^{\#}$ & $6.33 \pm 0.29^{\#}$ & 76.30 \\
\hline$R 25 Q$ & 0.023 & $444.13 \pm 7.17^{\#}$ & $130.40 \pm 4.72^{\#}$ & $3.4 \mathrm{I} \pm 0.07^{\#}$ & 41.13 \\
\hline FI64L & 0.023 & $679.03 \pm 3.60^{\#}$ & $94.5 I \pm 0.55$ & $7.19 \pm 0.07^{\#}$ & 86.70 \\
\hline$E 215 \mathrm{~K}$ & 0.047 & $720.67 \pm 7.66^{\#}$ & $89.00 \pm 2.10^{\#}$ & $8.10 \pm 0.11$ & 97.70 \\
\hline$F 219 S$ & 0.023 & $687.53 \pm 9.68^{\#}$ & $115.10 \pm 8.85$ & $5.99 \pm 0.37^{\#}$ & 72.19 \\
\hline V327M & 0.023 & $638.90 \pm 16.53^{\#}$ & $99.07 \pm 5.17$ & $6.45 \pm 0.17^{\#}$ & 77.91 \\
\hline D336N & 0.023 & $750.67 \pm 3.29^{\#}$ & $99.67 \pm 1.47$ & $7.53 \pm 0.09^{\#}$ & 90.85 \\
\hline V342M & 0.023 & $793.97 \pm 1.95^{\#}$ & II $0.90 \pm 0.87$ & $7.16 \pm 0.06^{\#}$ & 86.39 \\
\hline R344Q & 0.023 & $535.30 \pm 4.49^{\#}$ & $127.67 \pm 2.15^{\#}$ & $4.19 \pm 0.04^{\#}$ & 50.58 \\
\hline R440C & 0.023 & $231.77 \pm 1.36^{\#}$ & $65.5 \pm 2.59^{\#}$ & $3.54 \pm 0.13^{\#}$ & 42.64 \\
\hline R497C & 0.023 & $382.27 \pm 1.40^{\#}$ & $128.00 \pm 3.12^{\#}$ & $2.98 \pm 0.08^{\#}$ & 36.06 \\
\hline
\end{tabular}

Notes: Data are presented as the mean \pm standard deviation of three parallel experiments. Significantly different from the wild-type, ${ }^{\#} P<0.05$. Abbreviation: ND, not determined.

dapoxetine demethylation were altered in majority of these allelic variants $(2 D 6 * 2, * 10, * 87, * 88, * 90, * 91, * 93, * 94$, *95, *97, *98, *R25Q, F164L, F219S, V327M, D336N, $V 342 M, R 344 Q, R 440 C$, and $R 497 C$ ). Definitely, CYP2D6 allelic variants could be classified into categories, according to their intrinsic clearance values when compared with the counterpart of the wild-type (counted as $100 \%$ ): six variants $(2 D 6 * 2, * 10, * 93, R 25 Q, R 440 C$, and $R 497 C$ ) exhibited obviously decreased intrinsic clearance values $(20.44 \%-49.63 \%$ relative clearance); 14 alleles $(C Y P 2 D 6 * 87, * 88, * 90, * 91$, *94, *95, *97, *98, F164L, F219S, V327M, D336N, V342M, and $R 344 Q$ ) exhibited slight reduction $(50.58 \%-90.90 \%$ relative clearance). Another two variants $(2 D 6 * 89$ and $E 215 K)$ were without distinct difference $(92.81 \%$ and $97.70 \%$ relative clearance), according to the statistical analyses.

The results of the oxidative metabolism of dapoxetine are shown in Figure 3 and Table 2. Most of the tested allelic variants exhibited no significant changes on $\mathrm{V}_{\max }$ and/or $\mathrm{K}_{\mathrm{m}}$ values when compared with the counterparts of $2 D 6^{*} 1$ (count as $100 \%)$. Two variants $(2 D 6 * 93$ and $* 94)$ exhibited obviously increased $\mathrm{V}_{\max }$ values (124.95\%-135.58\%); five variants $(2 D 6 * 10, * 97, R 25 Q, E 215 K$, and $R 497 C)$ decreased to $33.26 \%-80.63 \%$. Four variants $(2 D 6 * 93, * 97, * 98$, and $D 336 N$ ) exhibited significantly increased $\mathrm{K}_{\mathrm{m}}$ values with $\sim 1.78$ - to 4.98 -fold as compared with $2 D 6^{*} 1$. Consequently, $\mathrm{V}_{\max } / \mathrm{K}_{\mathrm{m}}$ values for dapoxetine oxidation were altered in most of the tested allelic variants $(2 D 6 * 2, * 10, * 87, * 88$, *90, *91, *93, *94, *95, *97, *98, *R25Q, F164L, F219S, V327M, D336N, V342M, R344Q, R440C, R497C), except $2 D 6 * 90, * 94$, and $V 342 M$ without statistical significance. Compared with the $\mathrm{V}_{\max } / \mathrm{K}_{\mathrm{m}}$ value of $2 D 6^{*} 1$ (count as $100 \%$ ), eight variants $(2 D 6 * 10, * 93, * 95, * 97, * 98, R 25 Q, V 327 M$, and $R 497 C$ ) significantly declined to $27.56 \%-49.80 \%$; eleven alleles $(C Y P 2 D 6 * 2, * 87, * 88, * 89, * 91, F 164 L$, $E 215 K, F 219 S, D 336 N, R 344 Q$, and $R 440 C$ ) exhibited mild decreases $(50.34 \%-84.64 \%)$. Three variants $2 D 6^{*} 90$, $2 D 6^{*} 94$, and $V 342 M$ represented no significant increase (106.17\%-109.98\% relative clearance).

Furthermore, two defective alleles (CYP2D6*92 and CYP2D6*96) showed extremely low activity or no activity. Thus, the concentrations of both metabolites were below 
Table 2 Kinetic parameters from oxidational activities of recombinant wild-type and 24 CYP2D6 allelic variants toward dapoxetine

\begin{tabular}{|c|c|c|c|c|}
\hline Variants & $\begin{array}{l}V_{\max } \\
(\mathrm{pmol} / \mathrm{min} / \mathrm{nmol} \text { of } \mathrm{P} 450)\end{array}$ & $K_{m}(\mu M)$ & $\begin{array}{l}\text { Intrinsic clearance } \\
\left(\mathrm{V}_{\max } / \mathrm{K}_{\mathrm{m}}\right)\end{array}$ & $\begin{array}{l}\text { Relative clearance } \\
\text { (\% of wild-type) }\end{array}$ \\
\hline $2 D 6 * 1$ & $207.53 \pm 8.50$ & $633.67 \pm 41.26$ & $0.33 \pm 0.0 \mathrm{I}^{\#}$ & 100.00 \\
\hline 2D6*2 (R296C; S486T) & $185.47 \pm 12.00$ & $976.20 \pm 165.80$ & $0.19 \pm 0.019^{\#}$ & 58.45 \\
\hline 2D6*10 (P34S; S486T) & $69.03 \pm 1.96^{\#}$ & $713.83 \pm 35.61$ & $0.10 \pm 0.00^{\#}$ & 29.51 \\
\hline $2 \mathrm{D} 6 * 87(\mathrm{~A} 5 \mathrm{~V})$ & $180.00 \pm 7.32$ & $1,092.00 \pm 98.24$ & $0.17 \pm\left. 0.0\right|^{\#}$ & 50.34 \\
\hline $2 \mathrm{D} 6 * 88$ (VI04A) & $185.07 \pm 4.65$ & $728.57 \pm 22.52$ & $0.25 \pm 0.00^{\#}$ & 77.44 \\
\hline 2D6*89 (LI 42S) & $211.30 \pm 2.17$ & $909.57 \pm 35.66$ & $0.23 \pm 0.0 \mathrm{I}^{\#}$ & 70.87 \\
\hline $2 D 6 * 90(K / 47 R)$ & $235.63 \pm 2.90$ & $653.33 \pm 19.20$ & $0.36 \pm 0.01$ & 109.98 \\
\hline 2D6*9I (Cl6IS) & $|80.37 \pm 9.5|$ & $650.30 \pm 65.29$ & $0.28 \pm 0.00^{\#}$ & 84.64 \\
\hline 2D6*92 (2। 8frameshift) & ND & ND & ND & ND \\
\hline 2D6*93 (T249P) & $281.37 \pm 41.02^{\#}$ & $3,152.67 \pm 792.26^{\#}$ & $0.09 \pm 0.0 I^{\#}$ & 27.56 \\
\hline 2D6*94 (D337G) & $259.30 \pm 11.0 I^{\#}$ & $733.90 \pm 76.05$ & $0.35 \pm 0.02$ & 107.78 \\
\hline 2D6*95 (R388H) & $203.20 \pm 21.45$ & $\mathrm{I}, 263.67 \pm 206.4 \mathrm{I}$ & $0.16 \pm 0.0 I^{\#}$ & 49.26 \\
\hline 2D6*96 (424STOP) & ND & ND & ND & ND \\
\hline 2D6*97 (F457L) & $162.10 \pm 7.40^{\#}$ & $\mathrm{I}, 130.73 \pm 124.75^{\#}$ & $0.14 \pm\left. 0.0\right|^{\#}$ & 43.90 \\
\hline 2D6*98 (H463D) & $181.03 \pm 12.67$ & $\mathrm{I}, 462.33 \pm \mid 02.8 \mathrm{I}^{\#}$ & $0.12 \pm 0.00^{\#}$ & 37.73 \\
\hline$R 25 Q$ & $|44.77 \pm 4.7|^{\#}$ & $889.77 \pm 70.63$ & $0.16 \pm 0.0 I^{\#}$ & 49.80 \\
\hline FI64L & $211.27 \pm 17.17$ & $\mathrm{I}, 012.80 \pm 228.04$ & $0.21 \pm 0.03^{\#}$ & 65.14 \\
\hline$E 215 \mathrm{~K}$ & $167.33 \pm 6.80^{\#}$ & $826.27 \pm 48.18$ & $0.20 \pm 0.00^{\#}$ & 61.74 \\
\hline F219S & $206.80 \pm 6.92$ & $842.17 \pm 104.41$ & $0.25 \pm 0.02^{\#}$ & 75.18 \\
\hline V327M & $184.30 \pm 8.56$ & $1,254.00 \pm 169.27$ & $0.15 \pm 0.01^{\#}$ & 45.11 \\
\hline D336N & $232.80 \pm 7.88$ & $\mathrm{I}, 276.33 \pm \mathrm{I} \mid \mathrm{9} .40^{\#}$ & $0.18 \pm 0.0 I^{\#}$ & 55.82 \\
\hline V342M & $224.47 \pm 5.79$ & $646.37 \pm 65.4 \mathrm{I}$ & $0.35 \pm 0.03$ & 106.17 \\
\hline$R 344 Q$ & $|87.47 \pm| \mid .78$ & $935.20 \pm 143.54$ & $0.20 \pm 0.02^{\#}$ & 61.22 \\
\hline R440C & $191.17 \pm 7.27$ & $829.87 \pm 86.35$ & $0.23 \pm 0.02^{\#}$ & 70.45 \\
\hline R497C & II $5.30 \pm 4.05^{\#}$ & $995.10 \pm 75.38$ & $0.12 \pm 0.00^{\#}$ & 35.42 \\
\hline
\end{tabular}

Notes: Data are presented as the mean \pm standard deviation of three parallel experiments. Significantly different from the wild-type, ${ }^{\#}<<0.05$. Abbreviation: ND, not determined.

the detection limit and the kinetic parameters could not be determined.

\section{Discussion}

CYP2D6 is a highly polymorphic enzyme and is involved in the metabolism of many drugs. Nowadays, it participates in the metabolism of $\sim 30 \%$ of drugs in clinic. ${ }^{18}$
The polymorphism of CYP2D6 significantly affects the pharmacokinetics of its substrate drugs in clinic. ${ }^{19}$ The study of CYP2D6 polymorphism, which mainly cause drug metabolism differences and result in side effects, can provide references to the clinical research. There are significant differences among various racial and ethnic population on the frequency of CYP2D6 polymorphism..$^{20,21}$

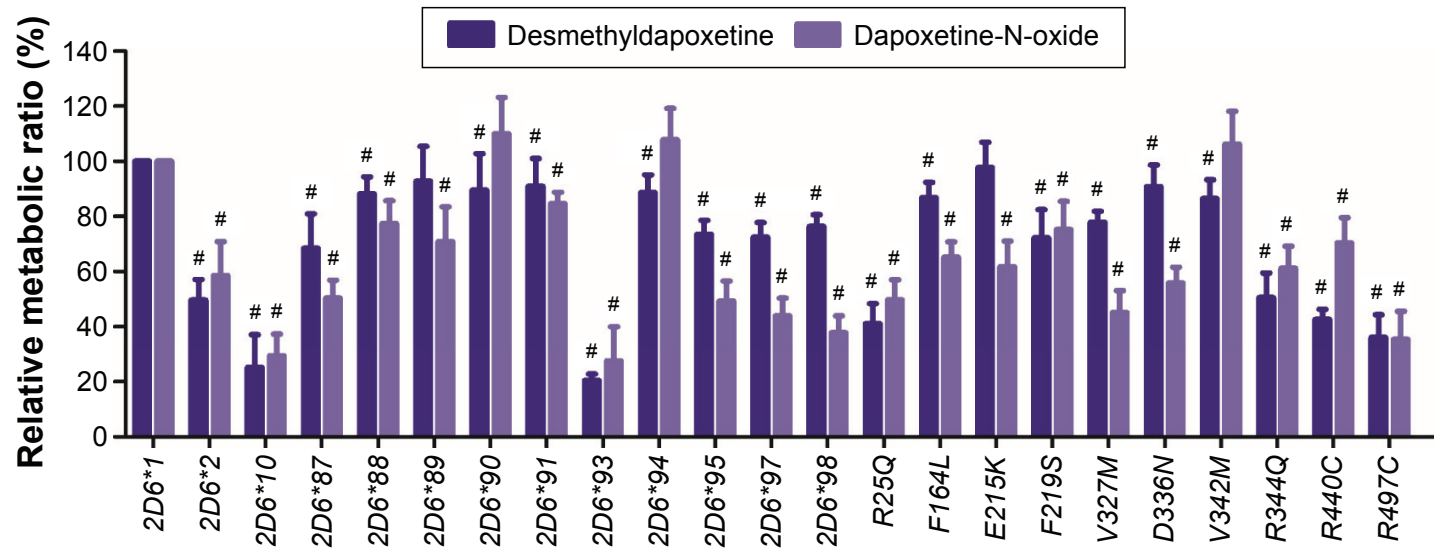

Figure 4 The catalytic activity of expressed CYP2D6 variants toward the two metabolites of dapoxetine, when compared with the counterpart values of the wild-type 2D6*l. Note: ${ }^{\#} P<0.05$. 
In our previous study, we performed a large-scale genetic investigation by sequencing the CYP2D6 gene in 2,129 healthy Chinese volunteers and 22 novel nonsynonymous variants were discovered and detected. ${ }^{7}$ This finding may greatly contribute to the development of personalized medicine for the Chinese Han population.

Dapoxetine has been recently evaluated for the treatment of PE by several countries. ${ }^{22,23}$ It is the only drug that has been approved for the on-demand treatment of PE. ${ }^{24}$ But in the process of treatment, a variety of adverse reactions occurred, so a considerable number of patients chose to spontaneously discontinue treatment with it. ${ }^{14}$ Thus, the effects of CYP2D6 polymorphism on dapoxetine metabolism in vitro have a great significance for the basic research and personalized treatment.

Two typical variants $C Y P 2 D 6^{*} 2$ and $C Y P 2 D 6^{*} 10$ were served as the positive controls for the functional analysis to ensure the reliability of this study. Recently, a study demonstrated that $C Y P 2 D 6 * 2$ exhibited a significantly decreased $\mathrm{V}_{\max } / \mathrm{K}_{\mathrm{m}}$ values for both bufuralol and dextromethorphan $\left(\sim 40 \%\right.$ of $\left.C Y P 2 D 6^{*} 1\right)$ with the baculovirus expression system. ${ }^{9}$ For $C Y P 2 D 6^{*} 10$, the most common allelic variant in oriental populations, several functional analyses have been conducted in various expression systems ${ }^{25}$ and it has higher $\mathrm{K}_{\mathrm{m}}$, lower $\mathrm{V}_{\max }$, and lower $\mathrm{V}_{\max } / \mathrm{K}_{\mathrm{m}}$ values for bufuralol, dextromethorphan, debrisoquine, atomoxetine, and nortriptyline in vitro. ${ }^{18}$ Our investigation revealed that both variants presented significantly decreased $\mathrm{V}_{\max } / \mathrm{K}_{\mathrm{m}}$ value on dapoxetine (approximately decreased by $50 \%$ for $C Y P 2 D 6 * 2$ and $75 \%$ for $C Y P 2 D 6^{*} 10$ ); these findings were consistent with the aforementioned studies.

To better understand the effects of CYP2D6 allelic variants on the metabolism of dapoxetine, we analyzed the 22 novel CYP2D6 variants in detail. In particular, three isoforms, $2 D 6^{*} 93, R 25 Q$, and $R 497 C$, exhibited significant changes on $\mathrm{V}_{\max } / \mathrm{K}_{\mathrm{m}}$ values, indicating that the amino acids in these sites have a vital impact on the metabolism of dapoxetine. For allelic isoform CYP2D6*93, the $\mathrm{V}_{\max } / \mathrm{K}_{\mathrm{m}}$ valve decreased by $70 \%$. For the other two variants, the $\mathrm{V}_{\max } / \mathrm{K}_{\mathrm{m}}$ valve of $R 497 C$ decreased by $\sim 65 \%$, while $R 25 Q$ decreased by more than $50 \%$. Furthermore, two allelic isoforms (CYP2D6*92 and CYP2D6*96) exhibited absent metabolic activity. CYP2D6*93 (Thr249Pro) contains one nucleotide substitution $(A>C)$ at position 745 in the complementary DNA (cDNA), which causes an amino acid change from Thr to Pro at position 249. ${ }^{8}$ The Thr-249 residue is adjacent to the residues Phe-247 and Leu-248, which are located on the border of CYP2D6 active site cavity, ${ }^{26}$ because Leu-248,
Leu-110, and Phe-112 constitute one access channel for substrate entrance. We speculate that the replacement of the hydrophilic Thr with the hydrophobic Pro at position 249 might influence the spatial structure of the adjacent egress channel and thus blocks the normal entrance of the CYP2D6 substrate.

For the isoform $R 25 Q$, which exhibited significantly decreased enzymatic activity on dapoxetine, it has been deduced that $\operatorname{Arg} 25$ is located within the transmembrane domain and acts as a halt transfer signal; thus, changes in this site might significantly decrease the enzymatic activity in vitro. ${ }^{27}$ For $R 497 C$, previous functional predictions revealed that this allelic isoform might deleteriously affect the CYP2D6 protein using bioinformation tools. ${ }^{7}$

Similar to the previously reported variants CYP2D6*20 (211 frameshift), CYP2D6*8 (Gly169STOP), CYP2D6*92 (218frameshift), and CYP2D6*96 (Gln424STOP) exhibited no enzymatic activity. ${ }^{1}$ CYP2D6*92 variant has a onenucleotide deletion (nucleotide C) at site 1995 in exon 4 and causes a frameshift effect: a disrupted reading frame and the premature termination of protein synthesis during its translation. CYP2D6*96 has a single-nucleotide mutation in exon 8 $\mathrm{C}>\mathrm{T}$ at position 3895 in the DNA (site 1270 in cDNA) and causes the codon 424 changing from CAG to one stop codon TAG. For $C Y P 2 D 6 * 92$ and $C Y P 2 D 6 * 96$, immunoblotting results revealed that both variants were expressed as truncated proteins, which caused the functional loss of enzymatic activity. ${ }^{8}$ We speculate that responsibility for the deficiency of enzymatic function might be the frameshift and resulting premature termination.

Particularly, the $\mathrm{V}_{\max } / \mathrm{K}_{\mathrm{m}}$ values of CYP2D6*89 and $E 215 \mathrm{~K}$ exhibited no difference compared with $2 D 6 * 1$ for demethylation, with $92.81 \%$ and $97.70 \%$, respectively. This phenomenon is not consistent with the previous research results.

For CYP2D6*89 (L142S), it contains one T to C substitution in site 1678 of the DNA sequence (425T $>C$ in the cDNA) and results in one amino acid change from Leu to Ser at position $142 .^{7}$ On the other hand, for $E 215 K$, Rowland et $\mathrm{a}^{26}$ found that many important amino acid residues were essential to the active site cavity. The Glu216 residue, which is located in the F helix, plays an important role in substrate recognition and binding. ${ }^{28}$ In the tested variants, $E 215 \mathrm{~K}$ and F219S were located in the F helix. ${ }^{7}$ Dai et al ${ }^{8}$ indicated that $C Y P 2 D 6^{*} 89$ and $E 215 K$ exhibited $>90 \%$ decrease in catalytic activity on bufuralol and dextromethorphan compared with the wild-type $C Y P 2 D 6 * 1 .{ }^{8}$ Cai et al ${ }^{9}$ reported that CYP2D6*89 decreased the metabolism of bufuralol, but had 
no effect on the metabolism of dextromethorphan in vitro; E215K exhibited $>97 \%$ decrease in catalytic activity on bufuralol and dextromethorphan.

As per our speculation, the different specificities of the substrate drug may be the main reason for this inconsistency. In another point of view, probing drugs cannot rule out some special factors in the process of metabolism of other drugs by one or two allelic variants.

In summary, we screened the enzymatic activity of the 24 variants of CYP2D6 on the metabolism of dapoxetine in vitro, especially the 22 novel isoforms. As the first report of all aforementioned alleles on dapoxetine metabolism, the research provided new information about CYP2D6 genetic polymorphism and related impact on its substrate drug dapoxetine; on the other hand, this study could help clinical assessment of the metabolic effects on dapoxetine, provide fundamental information to guide rational drug usage, and promote personalized medicine.

\section{Acknowledgments}

This work was supported by the Ministry of Health of the People's Republic of China (201302008), the First Affiliated Hospital of Wenzhou Medical University (FHY2015010), and a grant from the National Natural Science Foundation of China (No 31371280).

\section{Disclosure}

The authors report no conflicts of interest in this work.

\section{References}

1. Zhou SF, Liu JP, Chowbay B. Polymorphism of human cytochrome P450 enzymes and its clinical impact. Drug Metab Rev. 2009;41(2): 89-295.

2. Haufroid V, Hantson P. CYP2D6 genetic polymorphisms and their relevance for poisoning due to amphetamines, opioid analgesics and antidepressants. Clin Toxicol (Phila). 2015;53(6):501-510.

3. Susce MT, Murray-Carmichael E, de Leon J. Response to hydrocodone, codeine and oxycodone in a CYP2D6 poor metabolizer. Prog Neuropsychopharmacol Biol Psychiatry. 2006;30(7):1356-1358.

4. Okubo M, Murayama N, Miura J, Chiba Y, Yamazaki H. Effects of cytochrome P450 2D6 and 3A5 genotypes and possible coadministered medicines on the metabolic clearance of antidepressant mirtazapine in Japanese patients. Biochem Pharmacol. 2015;93(1):104-109.

5. Dapoxetine. Premature ejaculation: not worth the risk. Prescrire Int. 2010;19(105):12-14.

6. Cai WM, Nikoloff DM, Pan RM, et al. CYP2D6 genetic variation in healthy adults and psychiatric African-American subjects: implications for clinical practice and genetic testing. Pharmacogenomics J. 2006; 6(5):343-350.

7. Qian JC, Xu XM, Hu GX, et al. Genetic variations of human CYP2D6 in the Chinese Han population. Pharmacogenomics. 2013;14(14): $1731-1743$.
8. Dai DP, Geng PW, Wang SH, et al. In vitro functional assessment of 22 newly identified CYP2D6 allelic variants in the Chinese population. Basic Clin Pharmacol Toxicol. 2015;117(1):39-43.

9. Cai J, Dai DP, Geng PW, et al. Effects of 22 novel CYP2D6 variants found in the Chinese population on the bufuralol and dextromethorphan metabolisms in vitro. Basic Clin Pharmacol Toxicol. Epub 2015 Aug 27.

10. McCarty E, Dinsmore W. Dapoxetine: an evidence-based review of its effectiveness in treatment of premature ejaculation. Core Evid. 2012; 7:1-14.

11. Owen RT. Dapoxetine: a novel treatment for premature ejaculation. Drugs Today (Barc). 2009;45(9):669-678.

12. Jannini EA, Isidori AM, Aversa A, Lenzi A, Althof SE. Which is first? The controversial issue of precedence in the treatment of male sexual dysfunctions. J Sex Med. 2013;10(10):2359-2369.

13. Yue FG, Dong L, Hu TT, Qu XY. Efficacy of dapoxetine for the treatment of premature ejaculation: a meta-analysis of randomized clinical trials on intravaginal ejaculatory latency time, patient-reported outcomes, and adverse events. Urology. 2015;85(4):856-861.

14. Jern P, Johansson A, Piha J, Westberg L, Santtila P. Antidepressant treatment of premature ejaculation: discontinuation rates and prevalence of side effects for dapoxetine and paroxetine in a naturalistic setting. Int J Impot Res. 2015;27(2):75-80.

15. Andrews PW, Thomson JA Jr, Amstadter A, Neale MC. Primum non nocere: an evolutionary analysis of whether antidepressants do more harm than good. Front Psychol. 2012;3:117.

16. Kendirci M, Salem E, Hellstrom WJ. Dapoxetine, a novel selective serotonin transport inhibitor for the treatment of premature ejaculation. Ther Clin Risk Manag. 2007;3(2):277-289.

17. Modi NB, Dresser M, Desai D, Edgar C, Wesnes K. Dapoxetine has no pharmacokinetic or cognitive interactions with ethanol in healthy male volunteers. J Clin Pharmacol. 2007;47(3):315-322.

18. Shen H, He MM, Liu H, et al. Comparative metabolic capabilities and inhibitory profiles of CYP2D6.1, CYP2D6.10, and CYP2D6.17. Drug Metab Dispos. 2007;35(8):1292-1300.

19. Ingelman-Sundberg M. Genetic polymorphisms of cytochrome P450 2D6 (CYP2D6): clinical consequences, evolutionary aspects and functional diversity. Pharmacogenomics J. 2005;5(1):6-13.

20. Jaja C, Burke W, Thummel K, Edwards K, Veenstra DL. Cytochrome p450 enzyme polymorphism frequency in indigenous and native American populations: a systematic review. Community Genet. 2008;11(3): 141-149.

21. Weber WW. Populations and genetic polymorphisms. Mol Diagn. 1999;4(4):299-307.

22. Gur S, Sikka SC. The characterization, current medications, and promising therapeutics targets for premature ejaculation. Andrology. 2015; 3(3):424-442.

23. Giuliano F, Clement P. Pharmacology for the treatment of premature ejaculation. Pharmacol Rev. 2012;64(3):621-644.

24. Cormio L, Massenio P, La Rocca R, Verze P, Mirone V, Carrieri G. The Combination of dapoxetine and behavioral treatment provides better results than dapoxetine alone in the management of patients with lifelong premature ejaculation. $J$ Sex Med. 2015;12(7):1609-1615.

25. Hiratsuka $M$. In vitro assessment of the allelic variants of cytochrome P450. Drug Metab Pharmacokinet. 2012;27(1):68-84.

26. Rowland P, Blaney FE, Smyth MG, et al. Crystal structure of human cytochrome P450 2D6. J Biol Chem. 2006;281(11):7614-7622.

27. Sakuyama K, Sasaki T, Ujiie S, et al. Functional characterization of 17 CYP2D6 allelic variants (CYP2D6.2, 10, 14A-B, 18, 27, 36, 39, 47-51, 53-55, and 57). Drug Metab Dispos. 2008;36(12):2460-2467.

28. Guengerich FP, Hanna IH, Martin MV, Gillam EM. Role of glutamic acid 216 in cytochrome P450 2D6 substrate binding and catalysis. Biochemistry. 2003;42(5):1245-1253. 


\section{Publish your work in this journal}

Drug Design, Development and Therapy is an international, peerreviewed open-access journal that spans the spectrum of drug design and development through to clinical applications. Clinical outcomes, patient safety, and programs for the development and effective, safe, and sustained use of medicines are a feature of the journal, which

has also been accepted for indexing on PubMed Central. The manuscript management system is completely online and includes a very quick and fair peer-review system, which is all easy to use. Visit http://www.dovepress.com/testimonials.php to read real quotes from published authors.

Submit your manuscript here: http://www.dovepress.com/drug-design-development-and-therapy-journal 